\title{
Moist Convection and the Thermal Stratification of the Extratropical Troposphere
}

\author{
Tapio Schneider and Paul A. O'Gorman \\ California Institute of Technology, Pasadena, California
}

(Manuscript received 16 October 2007, in final form 11 April 2008)

\begin{abstract}
Simulations with an aquaplanet general circulation model show that sensible and latent heat transport by large-scale eddies influences the extratropical thermal stratification over a wide range of climates, even in relatively warm climates with small meridional surface temperature gradients. Variations of the lapse rate toward which the parameterized moist convection in the model relaxes atmospheric temperature profiles demonstrate that the convective lapse rate only marginally affects the extratropical thermal stratification in Earth-like and colder climates. In warmer climates, the convective lapse rate does affect the extratropical thermal stratification, but the effect is still smaller than would be expected if moist convection alone controlled the thermal stratification. A theory for how large-scale eddies modify the thermal stratification of dry atmospheres is consistent with the simulation results for colder climates. For warmer and moister climates, however, theories and heuristics that have been proposed to account for the extratropical thermal stratification are not consistent with the simulation results. Theories for the extratropical thermal stratification will generally have to take transport of sensible and latent heat by large-scale eddies into account, but moist convection may only need to be taken into account regionally and in sufficiently warm climates.
\end{abstract}

\section{Introduction}

The thermal stratification of the troposphere mediates many aspects of the response of climate to perturbations. For example, it determines the strength of the greenhouse effect and influences the energies and scales of the large-scale eddies that effect most of the transport of heat, mass, momentum, and water vapor in Earth's extratropical troposphere. Understanding the dynamic processes that control it is therefore essential for understanding the response of climate to perturbations. It has been known for some time that moist convection controls the thermal stratification in the tropics (e.g., Stone and Carlson 1979; Xu and Emanuel 1989; Emanuel et al. 1994). However, what dynamic processes control the thermal stratification in the extratropics is unclear.

In the zonal mean and particularly in winter, the extratropical troposphere is more stably stratified than a moist adiabat, indicating that heat fluxes associated with large-scale eddies play some role, and perhaps the

Corresponding author address: Tapio Schneider, California Institute of Technology, 1200 E. California Blvd., Pasadena, CA 91125-2300.

E-mail: tapio@caltech.edu dominant role, in setting the extratropical thermal stratification (Stone and Carlson 1979). Indeed, largescale eddies alone, interacting with radiative processes, can maintain an extratropical thermal stratification and tropopause similar to that of present-day Earth (Schneider 2004; Schneider and Walker 2006, hereafter SW06). On the other hand, air masses in some extratropical regions, for example, over midlatitude continents in summer and in storm tracks over oceans in winter, frequently exhibit thermal stratifications that are neutral or nearly neutral with respect to moist convection or slantwise moist convection (Emanuel 1988; Korty and Schneider 2007). This indicates that convective heat fluxes may also play a role in setting the extratropical thermal stratification, at least regionally.

Simulations with an idealized general circulation model (GCM) without a hydrologic cycle exhibit different climate regimes distinguishable according to whether the extratropical thermal stratification is controlled by (dry) convection or whether it is modified by large-scale eddies (SW06). Among other parameters, Schneider and Walker varied the lapse rate toward which the parameterized convection in the model relaxes atmospheric temperature profiles. For convective lapse rates statically more stable than dry adiabatic and for sufficiently small meridional surface temperature 
gradients, the extratropical thermal stratification was controlled by convection; for convective lapse rates close to or equal to dry adiabatic and/or for sufficiently large meridional surface temperature gradients, the extratropical thermal stratification was modified by largescale eddies. Here we pursue a strategy analogous to that in SW06 to assess whether a similar regime transition occurs in simulations with an idealized GCM with a hydrologic cycle. We vary the lapse rate toward which the parameterized moist convection in the model relaxes atmospheric temperature profiles to assess how moist convection affects the extratropical thermal stratification in a wide range of simulated climates. We also assess whether the heuristic proposed by Juckes (2000) and advocated by Frierson et al. (2006) and Frierson $(2006,2008)$ to account for the extratropical thermal and moisture stratification in convecting atmospheres is consistent with our simulation results.

\section{Simulations with aquaplanet GCM}

We use the aquaplanet GCM described in O'Gorman and Schneider (2008a), which resembles the GCM of Frierson et al. (2006). The GCM contains idealized representations of radiative transfer (gray atmosphere with prescribed longwave and shortwave optical depths) and moist thermodynamics (only the vapor-liquid phase transition is considered, and the latent heat of vaporization is taken to be constant). The lower boundary is a uniform slab ocean that does not transport heat, with fixed albedo and roughness. The forcing is temporally constant and zonally and hemispherically symmetric, so the simulated climates have stationary and zonally and hemispherically symmetric flow statistics.

Moist convection in the GCM is parameterized by a quasi-equilibrium scheme that relaxes temperatures in an atmospheric column toward a profile with lapse rate

$$
\Gamma_{c}=-\frac{\partial T_{c}}{\partial z}=\left\{\begin{array}{ccc}
\Gamma_{d} & \text { for } & z<z_{\mathrm{LCL}} \\
\gamma \Gamma_{m c} & \text { for } & z \geq z_{\mathrm{LCL}}
\end{array}\right.
$$

Below the lifted condensation level (LCL) of an air parcel lifted adiabatically from the lowest model level $(\sigma=0.99)$, the convective lapse rate $\Gamma_{c}$ is the dryadiabatic lapse rate $\Gamma_{d}$. Above the LCL, the convective lapse rate $\Gamma_{c}$ is the lapse rate $\gamma \Gamma_{m c}$, where $\gamma$ is a rescaling factor and $\Gamma_{m c}=\Gamma_{m c}(z)$ is the moist-adiabatic lapse rate (more precisely, the moist-pseudoadiabatic lapse rate) of a saturated parcel at temperature $T_{c}(z)$, with $T_{c}(z)$ obtained by integrating (1) from the lowest model upward. Convection occurs whenever there is sufficient positive convective available potential energy relative to the profile $T_{c}(z)$. Convection relaxes the specific hu- midity field toward a profile with constant relative humidity $(70 \%)$ relative to the profile $T_{c}(z)$. For deep convection, the uppermost level up to which convection adjusts the temperature and specific humidity is the level of neutral buoyancy corresponding to the profile $T_{c}(z)$; for shallow convection, it is a lower level. Conservation of the column-integrated moist enthalpy is ensured either by changing the time scale over which the specific humidity adjustment is made or by relaxing the temperature toward the shifted profile $T_{c}(z)+\Delta T_{c}$, with a suitably chosen $\Delta T_{c}$ that is constant with height in the convective part of each column. For $\gamma=1$, the profile $T_{c}(z)$ is a moist adiabat; the convection scheme is that described in Frierson (2007) with the modifications in O'Gorman and Schneider (2008a). For $\gamma<1$, the profile $T_{c}(z)$ is continuous with a dry adiabat at the LCL but above is statically more stable than the moist adiabat for $\gamma=1$; the convection scheme uses this rescaled profile $T_{c}(z)$ in calculations of convective criteria, precipitation rates, etc., in the same way in which the moist adiabat is used in the scheme for $\gamma=1$.

Moist convection in the GCM can also occur by resolved motions on the grid scale. However, the quasiequilibrium convection scheme in the GCM maintains a minimum static stability corresponding to the convective lapse rate. Moist convection on the model's grid scale therefore is suppressed in simulations with $\gamma<1$, in which the minimum static stability is greater than that of a moist adiabat.

For each value $\gamma=(0.8,0.9,1)$ of the convective rescaling factor, we generated a series of simulations by varying the optical thickness of an idealized longwave absorber. The longwave absorber may be thought of as representing the combined longwave effects of water vapor and well-mixed greenhouse gases, with shortwave absorption kept fixed as the longwave optical thickness is varied. The optical thickness was rescaled by factors $\alpha=(0.4,0.6,0.7,0.8,0.9,1.0,1.2,1.4,1.6,1.8$, $2.0,2.5,3.0,4.0)$ relative to the latitude-dependent optical thickness in a reference simulation $(\alpha=1)$, which has, for $\gamma=1$, an Earth-like climate. The series of simulations with $\gamma=1$ is a subset of that analyzed in O'Gorman and Schneider (2008a,b), encompassing climates with global-mean surface air temperatures between $270 \mathrm{~K}$ (pole-equator temperature contrast $63 \mathrm{~K}$ ) and $311 \mathrm{~K}$ (temperature contrast $25 \mathrm{~K}$ ). ${ }^{1}$ The reduced

\footnotetext{
${ }^{1}$ The simulations with $\alpha=0.2$ and 6.0 in O'Gorman and Schneider 2008a,b) are not included here because, for $\alpha=0.2$, the extratropical tropopause is poorly defined and, for $\alpha=6.0$ and $\gamma=0.8$, the tropical tropopause reaches the highest model levels, which would have made a change in vertical discretization necessary.
} 

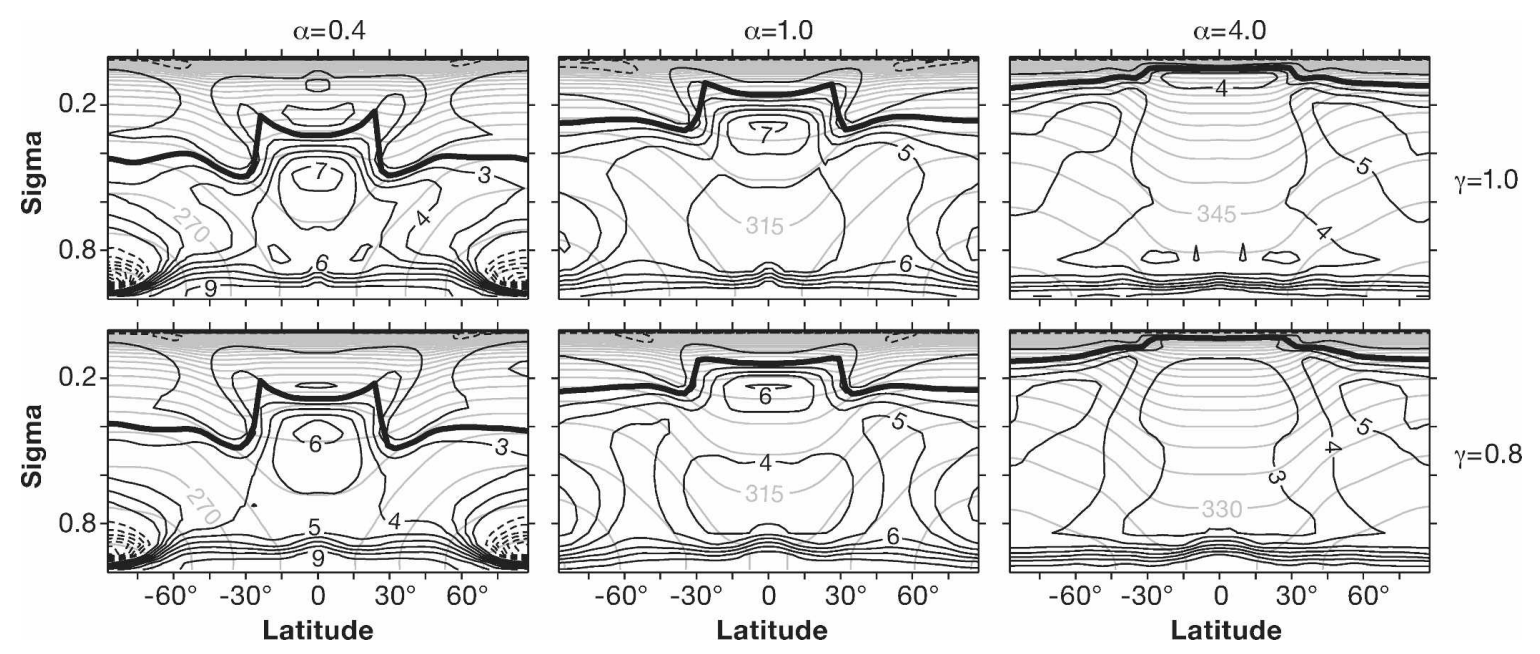

FIG. 1. Temperature lapse rate (black lines, contour interval $1 \mathrm{~K} \mathrm{~km}^{-1}$, negative contours dashed) and potential temperature (gray lines, contour interval $15 \mathrm{~K}$ ) for simulations with different optical-thickness rescaling factors $\alpha$ (columns) and different convective rescaling factors $\gamma$ (rows). The thick line marks the tropopause, identified as a $2 \mathrm{~K} \mathrm{~km}^{-1}$ isoline of temperature lapse rate. The vertical coordinate $\sigma=p / p_{s}$ is pressure $p$ normalized by surface pressure $p_{s}$.

convective rescaling factors $\gamma=(0.8,0.9)$ are small enough that they have a clear effect on the thermal stratification where moist convection controls it. Yet they are not so small that convection would generally become important for the thermal stratification where it is not important in simulations with $\gamma=1$, or that their expected effect on the thermal stratification and accompanying circulation changes in the tropics (e.g., expansion of the Hadley cell) would overly complicate the interpretation of changes in the extratropics.

The simulations were run with a spectral resolution of T42 in the horizontal and with 30 sigma levels in the vertical. To reach statistically steady states, the simulations for $\gamma=1$ were either spun up for 800 days from a slightly perturbed isothermal rest state or for 300 days from the end state of a simulation with similar longwave optical thickness. The simulations with $\gamma<1$ were spun up for 300 days from the corresponding simulation with $\gamma=1$. We will show zonal and temporal averages over a subsequent 200-day period of the simulations.

\section{Simulation results}

In each series of simulations with fixed convective rescaling factor $\gamma$, the surface temperature and tropopause height increase with increasing optical-thickness rescaling factor $\alpha$; the rows of Fig. 1 show examples. (The differences between the Northern and Southern Hemisphere in this and the following two figures are indicative of the sampling variability owing to the finiteness of the temporal average.) These increases were to be expected. The increase in surface temperature occurs because of the enhanced greenhouse effect, and the increase in tropopause height can also be understood from radiative considerations. For a semigray atmosphere (transparent to solar radiation and gray for longwave radiation) with an optically thin stratosphere-a qualitatively adequate idealization of the atmospheres in our simulations-the tropopause height $H_{t}$ is related to the surface temperature $T_{s}$, tropospheric temperature lapse rate $\Gamma$ (assumed constant), and emission height $H_{e}$ through

$$
H_{t} \approx(1-c) \frac{T_{s}}{\Gamma}+c H_{e}
$$

where $c=2^{-1 / 4} \approx 0.84$ (Schneider 2007). The increase in tropopause height thus occurs because of the increases in surface temperature and emission height with increasing $\alpha$, both, modulated by lapse rate changes, implying an increase in tropopause height. The lapse rate in the tropical free troposphere decreases with increasing $\alpha$, consistent with the decrease in the moistadiabatic lapse rate with increasing surface temperature and with relatively small changes in near-surface relative humidity (Held and Soden 2000; O'Gorman and Schneider 2008a). The lapse rate in the extratropical free troposphere, however, does not vary monotonically with $\alpha$ but takes on a maximum value (implying minimum static stability) near $\alpha=1$; see O'Gorman and Schneider (2008b) for a more detailed discussion of lapse rate changes with $\alpha$.

With decreasing $\gamma$ at fixed $\alpha$, the lapse rate in the tropical free troposphere decreases, consistent with moist convection controlling the thermal stratification there; the columns of Fig. 1 show examples. The tropi- 

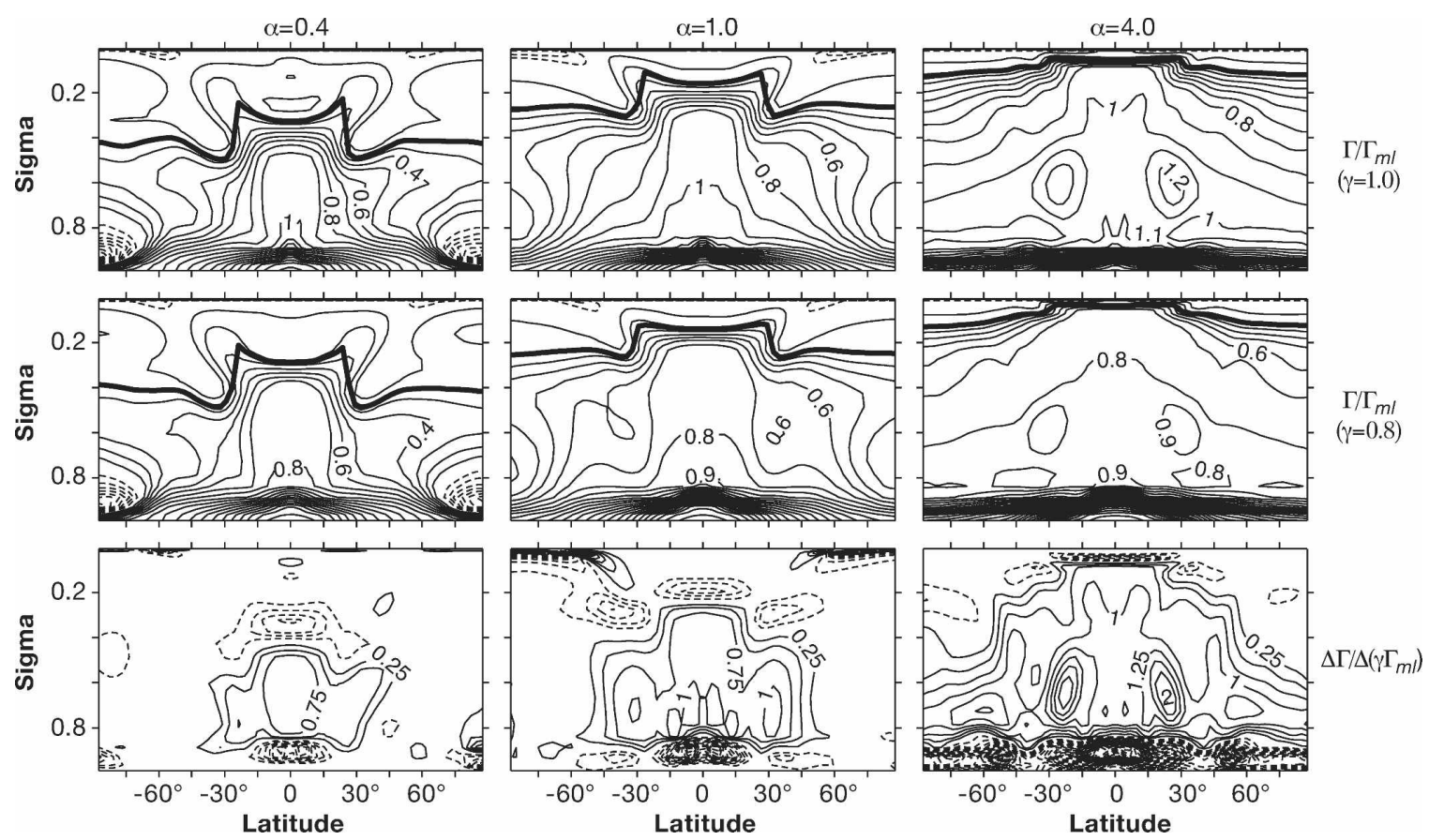

FIG. 2. (rows 1 and 2) Ratio of temperature lapse rate $\Gamma(\gamma)$ to moist-adiabatic lapse rate $\Gamma_{m l}(\gamma)$ for $\gamma=1$ and $\gamma=0.8$. (row 3) Change in temperature lapse rate $\Delta \Gamma=\Gamma(1)-\Gamma(0.8)$ normalized by $\Delta\left(\gamma \Gamma_{m l}\right)=\Gamma_{m l}(1)-0.8 \Gamma_{m l}(0.8)$. Here, the moist-adiabatic lapse rate $\Gamma_{m l}$ is the moist-adiabatic lapse rate calculated consistently with the idealized moist thermodynamics in the GCM from the local mean pressure and temperature. As in Fig. 1, columns correspond to different optical-thickness rescaling factors $\alpha$. The thick line marks the tropopause, and negative contours are dashed.

cal surface temperature also decreases because the strength of the greenhouse effect decreases along with the lapse rate, particularly for larger values of $\alpha$ for which the greater emission height implies a stronger effect of the lapse rate on the surface temperature (see the potential temperature contours in Fig. 1, particularly for $\alpha=4.0$ ). The changes in the extratropical thermal stratification are more subtle. In Earth-like and colder climates $(\alpha \lesssim 1)$, the lapse rate in the extratropical free troposphere poleward of the Hadley cells changes little if at all (see the columns for $\alpha=0.4$ and 1 in Fig. 1; the poleward boundaries of the Hadley cells in the upper troposphere are near the jumps in tropopause height). In warmer climates $(\alpha \gtrsim 1)$, the extratropical lapse rate changes more appreciably, but the changes, particularly in the upper troposphere, are still small compared with the tropical changes (see the column for $\alpha=4.0$ in Fig. 1).

Figure 2 quantifies how close the lapse rates are to moist adiabatic and how much they change with decreasing $\gamma$ in the simulations in Fig. 1. In the free troposphere of the deep tropics, above the LCL and planetary boundary layer $(\sigma \lesssim 0.85)$ and below the tropopause, the lapse rate $\Gamma$ is generally within $10 \%$ of the rescaled moist-adiabatic lapse rate $\gamma \Gamma_{m l}$, calculated consistently with the idealized moist thermodynamics in the GCM from the local mean pressure and temperature; that is, $\Gamma / \Gamma_{m l} \approx \gamma$ (see rows 1 and 2 of Fig. 2). Therefore, as $\gamma$ decreases from 1 to 0.8 at fixed $\alpha$, the decrease $\Delta \Gamma=\Gamma(1)-\Gamma(0.8)$ in lapse rate $\Gamma(\gamma)$ is approximately equal to the decrease $\Delta\left(\gamma \Gamma_{m l}\right)=$ $\Gamma_{m l}(1)-0.8 \Gamma_{m l}(0.8)$ in the rescaled moist-adiabatic lapse rate $\gamma \Gamma_{m l}(\gamma)$, or $\Delta \Gamma / \Delta\left(\gamma \Gamma_{m l}\right) \approx 1$ (see row 3 of Fig. 2). (Normalizing the lapse rate changes in this way takes into account the fact that the moist-adiabatic lapse rate can change as a result of atmospheric temperature changes with variations in $\gamma$, an effect that is significant in the warmest simulations.) Exceptions are regions near the tropopause, whose height generally increases as the lapse rate decreases, as implied by expression (2) for the tropopause height if decreases in surface temperature, as here, are relatively smaller than decreases in lapse rate [see also Held (1982) and Thuburn and Craig (2000) for similar radiative considerations]. These findings are what one would expect if the thermal stratification is controlled by moist convection.

In contrast, in the free troposphere of the extratropics, the lapse rate is generally substantially smaller than the rescaled moist-adiabatic lapse rate $\gamma \Gamma_{m l}$ in Earthlike and colder climates $(\alpha \lesssim 1)$-as in Earth's atmosphere in the zonal mean (Stone and Carlson 1979; Schneider 2007). In warmer climates $(\alpha \gtrsim 1)$, the lapse 

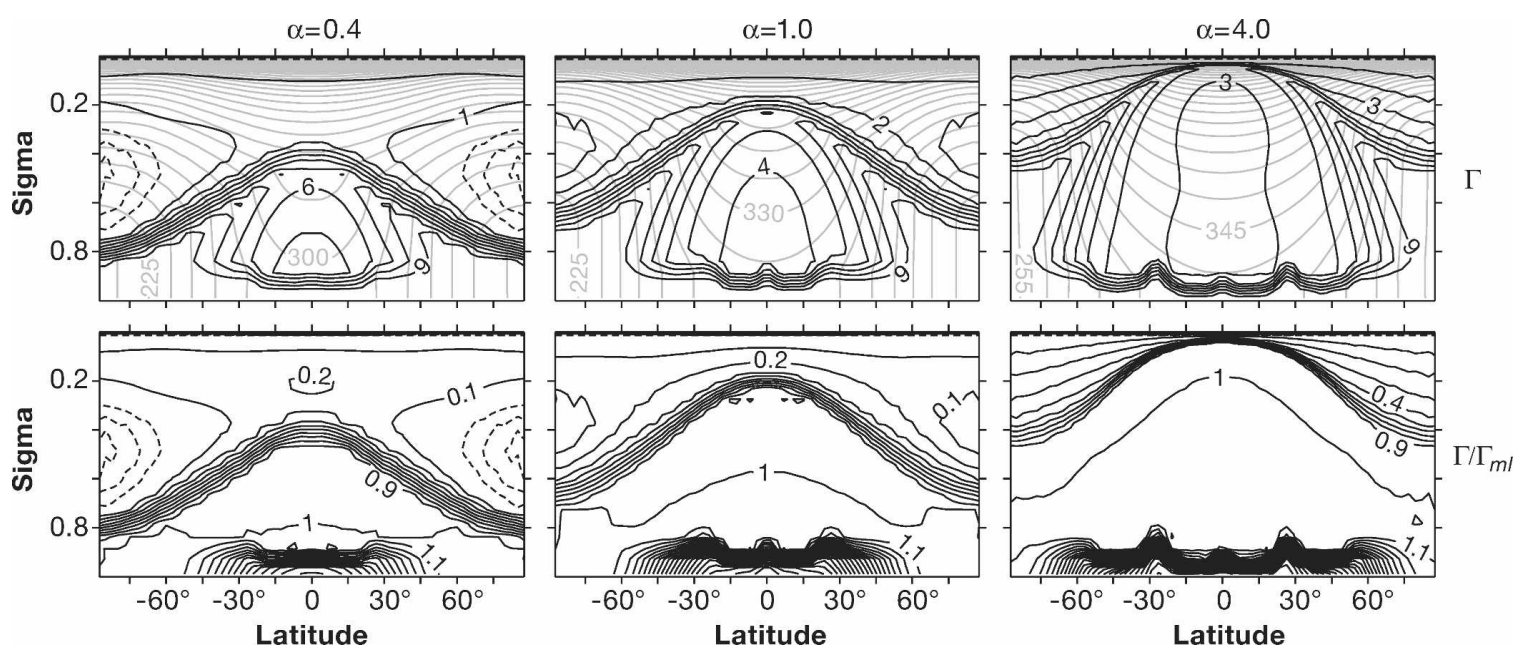

FIG. 3. (top row) As in row 1 of Fig. 1, temperature lapse rate (black lines, contour interval $1 \mathrm{~K} \mathrm{~km}^{-1}$, negative contours dashed) and potential temperature (gray lines, contour interval $15 \mathrm{~K}$ ) for radiative-convective equilibria with convective rescaling factor $\gamma=1$ and with different optical-thickness rescaling factors $\alpha$. (bottom row) As in row 1 of Fig. 2, ratio of temperature lapse rate $\Gamma$ to local moist-adiabatic lapse rate $\Gamma_{m l}$ for the radiative-convective equilibria in the top row. Shown are averages over 100 simulated days in the (oscillatory) radiative-convective equilibria.

rate is closer to $\gamma \Gamma_{m l}$ but comes to within about $10 \%$ of $\gamma \Gamma_{m l}$ only in the lower troposphere in the warmest simulations (see the column for $\alpha=4.0$ in Fig. 2). For $\alpha \lesssim 1$, with $\gamma$ decreasing from 1 to 0.8 , the normalized extratropical lapse rate decrease $\Delta \Gamma / \Delta\left(\gamma \Gamma_{m l}\right)$ poleward of about $50^{\circ}$ is less than 0.25 , with smaller decreases in higher latitudes. For $\alpha \gtrsim 1$, the normalized extratropical lapse rate decrease $\Delta \Gamma / \Delta\left(\gamma \Gamma_{m l}\right)$ poleward of about $50^{\circ}$ latitude can reach 1 in the lower troposphere but is smaller in the upper troposphere. It is evident that even in the warmest climates we simulated, the extratropical thermal stratification is not controlled by moist convection alone but remains influenced by large-scale dynamics. $^{2}$

To illustrate the effect large-scale dynamics have on

\footnotetext{
${ }^{2}$ The inferences from Fig. 2 are insensitive to the choice of reference lapse rate with which lapse rates are normalized. For example, in place of the local moist-adiabatic lapse rate $\Gamma_{m l}(\gamma)$, one can use as the reference lapse rate the convective lapse rate $\Gamma_{c}(\gamma)$, with the temporal- and zonal-mean temperature and specific humidity at the lowest model level as boundary condition. Because the extratropical free troposphere, in the temporal and zonal mean, is more stably stratified than the profile $\bar{T}_{c}(z)$ obtained by integrating (1) from mean surface conditions upward, the temperature $\bar{T}_{c}(z)$ at any given level is lower than the local mean temperature, and the convective lapse rate $\Gamma_{c}(\gamma)$ is greater than the rescaled local moist-adiabatic lapse rate $\gamma \Gamma_{m l}$. Therefore, the normalized lapse rate $\Gamma / \Gamma_{c}$ is smaller than $\Gamma /\left(\gamma \Gamma_{m l}\right)$ in the extratropical free troposphere, but only by less than $5 \%$ in the lower troposphere and by up to about $10 \%$ in the upper troposphere. The difference between the two normalized lapse rates is similarly small in the tropics, so that the normalized lapse rate change $\Delta \Gamma / \Delta\left(\Gamma_{c}\right)$ in the free troposphere does not differ substan-
}

the thermal stratification, Fig. 3 shows the lapse rate $\Gamma$ and the normalized lapse rate $\Gamma / \Gamma_{m l}$ in radiative-convective equilibria of the simulations with $\gamma=1$ in Figs. 1 and 2. The radiative-convective equilibria are simulated with the GCM by suppressing all lateral transport between atmospheric columns; because wind fields then are not predicted, fixed wind fields from the reference simulation are used as input to the surface flux and boundary layer schemes (O'Gorman and Schneider 2008a). Comparison of the first rows of Figs. 1 and 3 shows that large-scale dynamics (large-scale eddies and, particularly in the tropics, mean meridional circulations) reduce the pole-equator surface temperature contrast, increase the height of the extratropical tropopause, and reduce the slope of dry isentropes in the extratropical lower atmosphere. The second row of Fig. 3 shows that, above the LCL and planetary boundary layer and below the tropopause that forms in radiativeconvective equilibrium (recognizable by the rapid change in lapse rate), the lapse rate $\Gamma$ is close to the local moist-adiabatic lapse rate $\Gamma_{m l}$, implying that the radiative equilibria are statically unstable and are stabilized by convection. The convective layer is shallow in high latitudes, particularly in simulations with small

tially from the change $\Delta \Gamma / \Delta\left(\gamma \Gamma_{m l}\right)$ shown in Fig. 2. The same holds for the normalized lapse rate change $\Delta \Gamma / \Delta\left(\Gamma_{c}\right)$ if, in the calculation of the convective lapse rate $\Gamma_{o}$, the mean values plus one standard deviation of temperature and specific humidity at the lowest model level are used as a boundary condition, corresponding to a test of the hypothesis that only convection in anomalously warm and moist conditions controls the thermal stratification. 


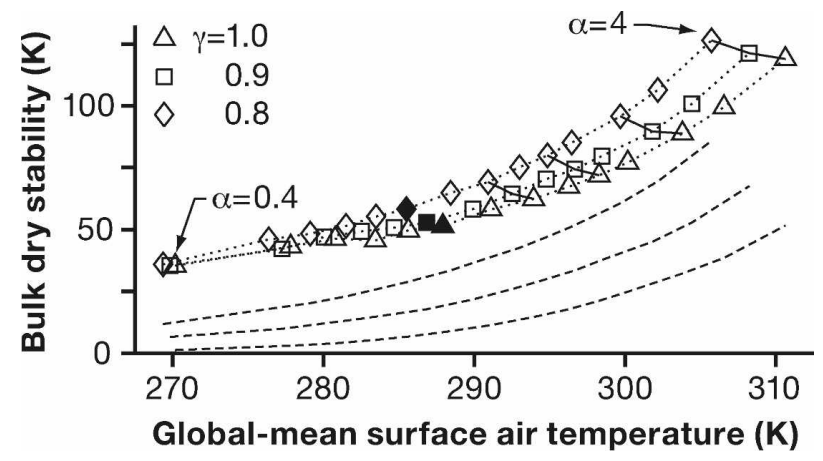

FIG. 4. Extratropical bulk dry stability (3) vs global-mean surface air temperature in a series of simulations with varying optical-thickness rescaling factor $\alpha$ and with convective rescaling factors $\gamma=(0.8,0.9,1)$. The set of rescaling factors $\alpha=(0.4, \ldots, 4)$ is the same in the three series of simulations. Solid lines connect selected simulations with the same $\alpha$, and filled symbols identify the simulations with $\alpha=1$. The dashed lines represent convective bulk stabilities, with $\gamma=0.8,0.9$, and 1.0 from top to bottom.

longwave optical thickness. Where temperatures and specific humidities are low, the convective lapse rate is close to the dry-adiabatic lapse rate $9.8 \mathrm{~K} \mathrm{~km}^{-1}$. The convection scheme with $\gamma=1$ approaches a dry convection scheme in the limit of vanishing specific humidity. In high latitudes in some of the radiative-convective equilibrium simulations with $\gamma=1$, the dry convection is performed by the boundary layer scheme rather than the convection scheme; however, in radiative-convective equilibrium simulations with $\gamma<1$, the convection scheme is active at all latitudes and maintains the more stable rescaled convective lapse rate. Comparison of Fig. 3 with Figs. 1 and 2 makes explicit that large-scale dynamics influence the extratropical thermal stratification.

Figure 4 confirms that large-scale dynamics influence the extratropical thermal stratification over the entire range of climates we simulated. It shows the extratropical bulk (dry) stability

$$
\Delta_{d}=\left\langle\bar{\theta}_{t}-\bar{\theta}_{s}\right\rangle
$$

as a function of the global-mean surface air temperature, where the overbar $\overline{(\cdot)}$ denotes a temporal and zonal mean and $\langle\cdot\rangle$ denotes an area-weighted average over a baroclinic zone, taken between $40^{\circ}$ and $70^{\circ}$ latitude in both, statistically identical, hemispheres. (The maximum meridional extent of the Hadley cell in the simulations is $33^{\circ}$ latitude, so the baroclinic zones lie poleward of the Hadley cells and immediately adjacent latitude zones.) The tropopause potential temperature $\bar{\theta}_{t}$ is determined by cubic spline interpolation as the potential temperature at the $2 \mathrm{~K} \mathrm{~km}^{-1}$ isoline of temperature lapse rate identified with the tropopause (see
Figs. 1 and 2); the near-surface potential temperature $\bar{\theta}_{s}$ is evaluated at the level $\sigma=0.84$, which, in the extratropics in all simulations, is above or near the top of the planetary boundary layer and above the LCL implied by the temporal- and zonal-mean temperature and specific humidity at the lowest model level. Figure 4 shows that the bulk stability increases with $\alpha$-primarily because the tropopause height increases-but that it only increases appreciably with decreasing $\gamma$ at fixed $\alpha$ in warmer climates. It also shows that the global-mean surface air temperature decreases with decreasing $\gamma$, in part because the strength of the greenhouse effect decreases and in part because of dynamical effects (e.g., see the changes in the boundary layer in Fig. 2). The dashed lines show convective bulk stabilities, for each simulation averaged over latitude zones in the same way as the bulk stability in the simulation. ${ }^{3}$ This is the bulk stability that would be expected if moist convection alone controlled the extratropical thermal stratification. The bulk stability in all simulations is considerably greater than the convective bulk stability. Quantitative details depend on how the average over baroclinic zones is defined, how the tropopause is identified, and how much regions of increased static stability near the tropopause (see Figs. 1 and 2) contribute to the bulk stability, which can lead to bulk stabilities exceeding convective bulk stabilities even in the deep tropics. However, regardless of whether one uses averages over baroclinic zones that move with the maximum of the near-surface eddy potential temperature flux as in SW06, for example, or evaluates bulk stabilities for subsets of the simulations between a nearsurface level and a fixed upper-tropospheric level, the results do not change qualitatively. The central conclusion is robust: the extratropical thermal stratification is statically significantly more stable than would be expected if moist convection alone controlled it.

Over the entire range of climates simulated, then, the extratropical thermal stratification is modified by largescale dynamics-by the eddies that primarily effect the large-scale heat transport in the extratropics. The eddy heat transport consists of slanted (poleward-upward)

\footnotetext{
${ }^{3}$ The convective bulk stability for a given latitude and simulation is determined from the profile $\bar{T}_{c}$ obtained by integrating the convective lapse rate (1) upward, with the temporal- and zonalmean temperature and specific humidity at the lowest model level as a boundary condition. The temporal- and zonal-mean tropopause pressure from the simulation is used to determine, by cubic spline interpolation, the tropopause potential temperature $\bar{\theta}_{c t}$ from $\bar{T}_{c}$; the near-surface potential temperature $\bar{\theta}_{c s}$ is again evaluated at the level $\sigma=0.84$. The convective bulk stabilities $\bar{\theta}_{c t}-\bar{\theta}_{c s}$ are then averaged between $40^{\circ}$ and $70^{\circ}$ latitude in both hemispheres, as for the bulk stability (3).
} 
fluxes of sensible and latent heat, where the relative importance of the latent heat transport increases as the climate warms (O'Gorman and Schneider 2008a). Thus, transport of latent heat by large-scale eddies and its release in large-scale phase changes of water must generally be taken into account in theories of the extratropical thermal stratification. In our aquaplanet simulations, however, it appears that moist convection needs to be taken into account only in sufficiently warm climates.

\section{Theories and heuristics}

\section{a. Supercriticality constraint for dry atmospheres}

SW06 showed that large-scale averages of the extratropical thermal structures of dry atmospheres satisfy the constraint that the supercriticality

$$
S_{c}=-\frac{f_{0}}{\beta_{0}} \frac{\left\langle\partial_{y} \bar{\theta}_{s}\right\rangle}{\Delta_{v}}
$$

a measure of the slope of isentropes, does not substantially exceed one. Here, $f_{0}$ and $\beta_{0}$ are reference values of the Coriolis parameter and of its derivative, $\partial_{y} \bar{\theta}_{s}$ is a surface or near-surface potential temperature gradient, and the bulk stability

$$
\Delta_{v}=-2\left\langle{\overline{\partial_{p} \theta}}^{s}\right\rangle\left\langle\bar{p}_{s}-\bar{p}_{t}\right\rangle,
$$

with surface pressure $\bar{p}_{s}$ and tropopause pressure $\bar{p}_{t}$, involves a static stability near the surface, $-\bar{\partial}_{p} \theta$, with $\overline{(\cdot)}^{s}$ denoting a near-surface temporal and zonal mean. The bulk stability (5) generally differs from and does not scale linearly with the bulk stability (3) considered above. When the tropopause height is somewhat greater than the scale height and the lapse rate does not vary strongly in the vertical within the free troposphere, the two bulk stabilities are approximately equal; when the tropopause height is much greater than the scale height, as in the warmer climates we simulated, the bulk stability (5) is generally smaller than the bulk stability (3) (see SW06, their appendix A).

In the dry GCM simulations in SW06, the supercriticality satisfied $S_{c}<1$ when convection controlled the extratropical thermal stratification and $S_{c} \sim 1$ when large-scale eddies modified the extratropical thermal stratification. Figure 5 shows that in our moist GCM simulations, the supercriticality still satisfies $S_{c} \lesssim 1$. $^{4}$ The supercriticality satisfies $S_{c} \sim 1$ in colder climates

\footnotetext{
${ }^{4}$ In Fig. 5, the average $\langle\cdot\rangle$ is taken between $40^{\circ}$ and $70^{\circ}$ latitude in both hemispheres as before, and $f_{0} / \beta_{0}$ is evaluated at $45^{\circ}$ latitude, which, in the simulations, is close to the latitude of maximum meridional eddy flux of near-surface potential temperature (cf. SW06). (The maximum of the eddy potential temperature flux at
}

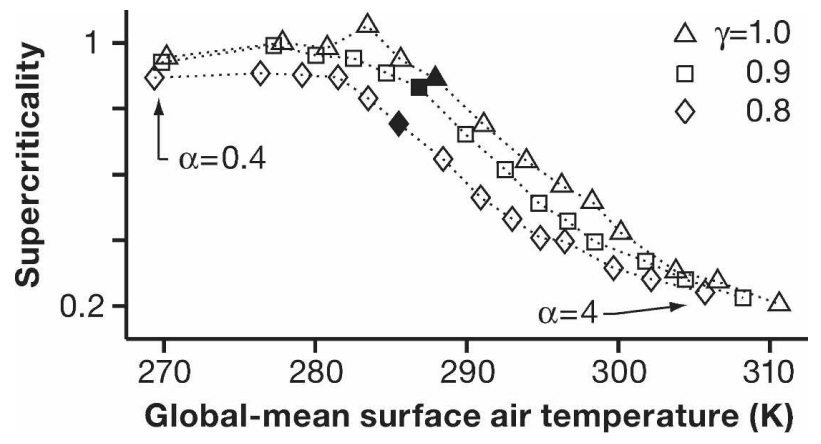

FIG. 5. Supercriticality (4) vs global-mean surface air temperature. As in Fig. 4, filled symbols identify the simulations with $\alpha=1$.

( $\alpha \lesssim 0.8)$ and decreases with increasing $\alpha$ in warmer climates $(\alpha \gtrsim 1)$. It decreases both because the meridional potential temperature gradient near the surface decreases with increasing $\alpha$ (O'Gorman and Schneider $2008 \mathrm{~b}$ ) and because the bulk stability (5) increases with increasing $\alpha$ for $\alpha \gtrsim 1$. The extratropical thermal structures in the GCM simulations examined in Frierson (2008) likewise appear to satisfy $S_{c} \lesssim 1$, provided the different bulk stability measures used there are similar to the bulk stability (5).

The constraint $S_{c} \lesssim 1$ for dry atmospheres follows from an estimate of the pressure range over which large-scale eddies stabilize the thermal stratification by redistributing entropy from the surface into the interior atmosphere. The zonal momentum or potential vorticity balance in isentropic coordinates implies a balance condition between geostrophic mass fluxes associated with the planetary-geostrophic eddy flux of potential vorticity along isentropes and with the geostrophic eddy flux of surface potential temperature (Schneider $2005,2007)$. If one assumes that the eddy fluxes can be

$\sigma=0.84$ lies between $42^{\circ}$ and $49^{\circ}$ latitude in all simulations but the four with $\alpha \geq 2$ and $\gamma=1$, in which it lies farther poleward and reaches up to $55^{\circ}$ latitude.) The potential temperature gradient $\partial_{y} \bar{\theta}_{s}$ near the surface is evaluated at $\sigma=0.84$, and the static stability $-\bar{\partial}_{p} \theta$ is evaluated from the derivative of potential temperature with respect to height $\frac{\partial_{z} \theta}{s}$ at $\sigma=0.84$, using the density at the lowest model level to obtain $-{\overline{\partial_{p}}}^{s}$ as in SW06. Different averaging conventions can yield slightly different supercriticalities, but the general behavior is robust: the supercriticality decreases from $O(1)$ values in colder climates to small values in warm climates (provided $f_{0} / \beta_{0}$ is evaluated near or at the maximum of the nearsurface eddy potential temperature flux and other quantities are averaged at least over eddy length scales). For example, using averages $\langle\cdot\rangle$ that move with the maximum of the near-surface eddy potential temperature flux as in SW06 yields supercriticalities that can be larger than the ones in Fig. 5, reaching about 1.2 in the coldest simulation; however, they show the same general behavior. 
modeled as diffusive fluxes with an eddy diffusivity that has no essential vertical structure and makes additional approximations, for example, to relate isentropic-coordinate mean fields to Eulerian mean fields, one obtains the estimate

$$
\left\langle\bar{p}_{s}-\bar{p}_{e}\right\rangle \sim\left\langle\bar{p}_{s}-\bar{p}_{t}\right\rangle S_{c}
$$

for the mean pressure difference between the surface $\left(\bar{p}_{s}\right)$ and the level $\left(\bar{p}_{e}\right)$ up to which large-scale eddy entropy fluxes extend (SW06; Schneider 2007). The constraint $S_{c} \lesssim 1$ then follows because what characterizes the tropopause is that it is the top of the atmospheric layer within which entropy received at the surface is redistributed, such that $\bar{p}_{e} \gtrsim \bar{p}_{t}$. See SW06 and Schneider and Walker (2008) for implications of the constraint $S_{c} \lesssim 1$ for the nature of atmospheric macroturbulence.

The arguments leading to the constraint $S_{c} \lesssim 1$ do not necessarily carry over to moist atmospheres but may provide adequate heuristics at low surface temperatures and thus at low specific humidities. Indeed, in our simulations for colder climates, $S_{c} \sim 1$ and largescale eddies modify the extratropical thermal stratification throughout the troposphere (e.g., Figs. 1-3, left column). This is consistent with the estimate (6) for the pressure range over which large-scale eddy fluxes of dry entropy extend. We expect that latent heat fluxes by large-scale eddies do not extend to higher levels than dry entropy fluxes, so a constraint on the vertical extent of dry entropy fluxes may still constrain the thermal stratification of moist atmospheres. However, in the simulations for warmer climates, $S_{c} \ll 1$ although largescale eddies modify the extratropical thermal stratification up to near the tropopause (e.g., Figs. 1-3, right column). Visual inspection of fields such as the vertical eddy flux of potential temperature also indicates that substantial large-scale eddy fluxes of dry entropy extend to near the tropopause (to within $100 \mathrm{hPa}$ of it or less), even in the warmest climates we simulated. Thus, the estimate (6) for the pressure range over which large-scale eddy fluxes of dry entropy extend is inadequate in the warmer climates. Estimates based on baroclinic-adjustment hypotheses (e.g., Stone 1972, 1978; Held 1982), which have a similar functional form as the estimate (6), suffer from similar inadequacies, in addition to the fundamental problem that our simulated atmospheres generally are baroclinically unstable and not neutral in the mean (T. Merlis 2008, personal communication).

Given that the estimate (6) derives from the zonal momentum or potential vorticity balance, which is not directly affected by moist processes, the reasons for its inadequacy in warmer and moister climates are not ob- vious. There are likely several reasons. For example, latent heat release can affect the vertical structure of the eddy diffusivities of potential vorticity and surface potential temperature or can lead to inadequacy of diffusive eddy flux closures, resulting in modifications of the estimate (6). Likewise, changes in the vertical structure of the geostrophic mass flux along near-surface isentropes with increasing surface temperature appear to affect the relevant value of the near-surface isentropic density or inverse static stability in the balance condition on eddy fluxes from which the estimate (6) derives (Schneider 2005); this results in inaccuracies of the estimate (6) that are independent of closure assumptions for eddy fluxes. An analysis of how moist processes modify the vertical structure of the mass and eddy fluxes along isentropes will be necessary to find ways to generalize the estimate (6) to moist atmospheres.

\section{b. Convection and large-scale eddies in moist atmospheres}

Juckes (2000) proposed a heuristic to account for the effect of large-scale eddies and moist convection on the combined extratropical thermal and moisture stratification, postponing questions of how the moisture distribution (relative humidity) is determined. He proposed that the bulk moist stability

$$
\Delta_{e}=\left\langle\bar{\theta}_{e t}^{*}-\bar{\theta}_{e s}\right\rangle
$$

may be representable as the sum of the minimum bulk moist stability $\Delta_{e c}$ maintained by moist convection and a multiple of the standard deviation $\left\langle\operatorname{std}\left(\theta_{e t}^{*}-\theta_{e s}\right)\right\rangle$ of instantaneous bulk moist stabilities $\theta_{e t}^{*}-\theta_{e s}$ :

$$
\Delta_{e} \approx \Delta_{e c}+d^{\prime}\left\langle\operatorname{std}\left(\theta_{e t}^{*}-\theta_{e s}\right)\right\rangle .
$$

Here, $d^{\prime}$ is an empirical constant, $\theta_{e t}^{*}$ is the saturation equivalent potential temperature at the mean level of the tropopause or at a fixed upper-tropospheric level, ${ }^{5}$ and $\theta_{e s}$ is the equivalent potential temperature at the surface. Following Frierson et al. (2006), at the upper level we use the saturation equivalent potential temperature, in place of the equivalent potential temperature used by Juckes (2000), to obtain a bulk moist stability (7) that vanishes for the temperature profile of a parcel lifted adiabatically from the surface, irrespective of the actual relative humidity at the upper level. If

\footnotetext{
${ }^{5}$ Note that $\theta_{e t}^{*}$ is not the saturation equivalent potential temperature at the instantaneous tropopause but at the mean level of the tropopause (or at a fixed upper-tropospheric level). The latter Eulerian quantity is needed to obtain an Eulerian mean bulk moist stability.
} 

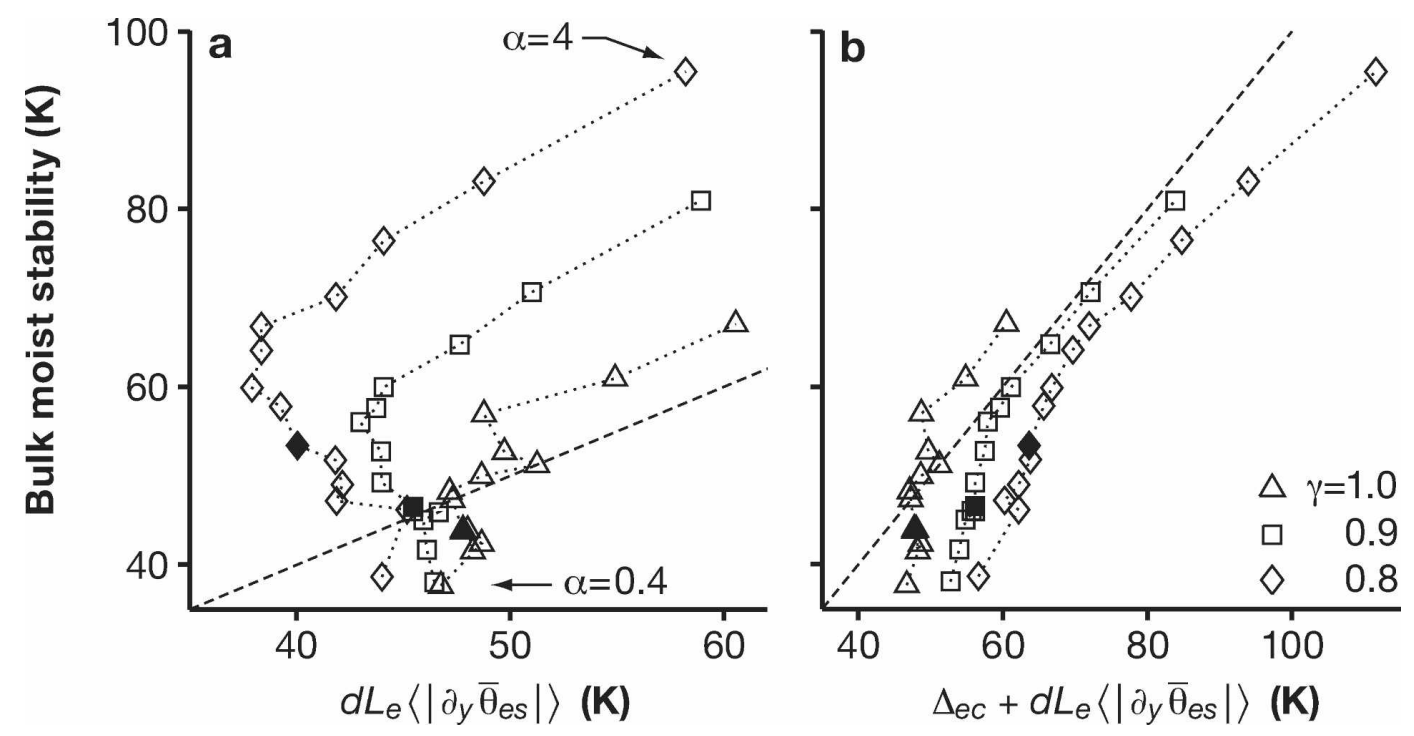

FIG. 6. Extratropical bulk moist stability between surface and tropopause vs (a) $d L_{e}|| \partial_{y} \bar{\theta}_{e s}|\rangle$ and (b) $\Delta_{e c}+$ $d L_{e}\left\langle\left|\partial_{y} \bar{\theta}_{e s}\right|\right\rangle$, with $d L_{e}=0.75 \mathrm{a}$. The results for $\gamma=1$ in (a) and (b) are identical because $\Delta_{e c} \equiv 0$. The dashed lines are the unit diagonals (note the different scales of the horizontal axes). As in Fig. 4, filled symbols identify the simulations with $\alpha=1$.

convection maintains a minimum static stability corresponding to such a moist-adiabatic temperature profile, as with our convection scheme for $\gamma=1$, the minimum bulk moist stability $\Delta_{e c}$ is zero, but it is greater than zero with our convection scheme for $\gamma<1$. A representation of the mean bulk moist stability of the form (8) is adequate if the instantaneous bulk moist stabilities follow, for example, an exponential distribution, but the mean of a distribution cannot generally be represented as a minimum plus a constant multiple of the standard deviation.

From the representation (8) of the mean bulk moist stability, Juckes obtained a bulk moist stability estimate in terms of mean-flow quantities by using mixing-length arguments. Assuming that

(i) the standard deviation $\left\langle\operatorname{std}\left(\theta_{e t}^{*}-\theta_{e s}\right)\right\rangle$ of bulk moist stabilities is either dominated by fluctuations in surface equivalent potential temperature $\theta_{e s}$, or fluctuations in upper-level saturation equivalent potential temperature $\theta_{e t}^{*}$ covary linearly with fluctuations in surface equivalent potential temperature $\theta_{e s}$, such that $\left\langle\operatorname{std}\left(\theta_{e t}^{*}-\theta_{e s}\right)\right\rangle \propto\left\langle\operatorname{std}\left(\theta_{e s}\right)\right\rangle$; and

(ii) the standard deviation of surface equivalent potential temperatures can be represented as $\left\langle\operatorname{std}\left(\theta_{e s}\right)\right\rangle \propto$ $L_{e}\left\langle\left|\partial_{y} \bar{\theta}_{e s}\right|\right\rangle$, where $L_{e}$ is an eddy length scale and $\partial_{y} \bar{\theta}_{e s}$ is the meridional equivalent potential temperature gradient at the surface,

one obtains the bulk moist stability estimate

$$
\Delta_{e} \approx \Delta_{e c}+d L_{e}\left\langle\left|\partial_{y} \bar{\theta}_{e s}\right|\right\rangle
$$

where $d$ is a new empirical constant. This is the estimate that Frierson et al. (2006) and Frierson (2006, 2008) advocated as accounting for the extratropical thermal and moisture stratification in simulations with idealized and comprehensive GCMs. It differs from the estimate originally proposed by Juckes in that the equivalent potential temperature gradient is evaluated at the surface rather than at a midtropospheric level, reflecting assumption (i) that the standard deviation of bulk moist stabilities is controlled by near-surface dynamics. In the low-temperature limit, in which equivalent potential temperatures approach dry potential temperatures, the bulk moist stability estimate (9) for ordinary convection $\left(\Delta_{e c}=0\right)$, with the tropopause as the upper level, formally resembles the estimate for the bulk dry stability (5) implied by $S_{c} \sim 1$ but with the length scale $d L_{e}$ replacing $f_{0} / \beta_{0}$. See Juckes (2000) and Schneider (2007) for further discussion and an assessment of the consistency of the estimate (9) with seasonal variations of the extratropical thermal and moisture stratification in Earth's atmosphere.

Our simulations allow us to test the bulk moist stability estimate (9) in a wide range of climates and to test the underlying assumptions about the effect of moist convection by varying the minimum bulk moist stability $\Delta_{e c}$ through variation of the convective rescaling factor $\gamma$. Figure 6 shows the extratropical bulk moist stability (7) between the surface and the tropopause as a function of the estimate (9), with $\Delta_{e c}=0$ in Fig. 6a and with the convective bulk stability $\Delta_{e c}>0$ associated with the 
rescaled convective lapse rate for $\gamma=0.9$ and 0.8 in Fig. 6 b. ${ }^{6}$ The equivalent potential temperature gradient $\partial_{y} \bar{\theta}_{e s}$ in the estimate (9) is evaluated at the lowest model level because this leads to better fits than evaluating it at other near-surface levels. The results are sensitive to this choice: the equivalent potential temperature gradient near the surface depends strongly on the level at which it is evaluated, with its absolute value generally decreasing with height because of the strong decrease of specific humidity with height. The length scale $L_{e}$ is chosen to be constant because the energy-containing spherical wavenumber of barotropic eddy kinetic energy varies only between 6.7 and 8.5 in the simulations. The constant $d L_{e}=0.75 a$, with Earth's radius $a$, is estimated to minimize the squared deviations of the bulk moist stabilities from the estimate (9) for the series of simulations with $\gamma=1$.

It is evident from Fig. 6 that the estimate (9) is not consistent with the bulk moist stability variations in the simulations. The bulk moist stability generally increases with increasing $\alpha$. However, in colder and moderately warm climates (e.g., $\alpha \lesssim 1.6$ for $\gamma=1$ ), the equivalent potential temperature gradient at the lowest model level does not increase correspondingly (Fig. 6a); in fact, it does not vary substantially as $\alpha$ increases because decreases in the dry potential temperature gradient are approximately compensated by increases in the specific humidity gradient. In warm climates (e.g., $\alpha \gtrsim$ 1.8 for $\gamma=1.0)$, the bulk moist stability increases roughly linearly with the equivalent potential temperature gradient at the lowest model level, with different intercepts for different $\gamma$. However, taking the variations of the convective lapse rate with $\gamma$ into account by including the convective bulk moist stability $\Delta_{e c}>0$ for $\gamma<1$ in the estimate (9) overcompensates for the different intercepts in warm climates (Fig. 6b). It also leads to overestimation of bulk moist stability variations in cold climates, consistent with the inferences from Fig. 2.

\footnotetext{
${ }^{6}$ In Fig. 6 , the average $\langle\cdot\rangle$ is again taken between $40^{\circ}$ and $70^{\circ}$ latitude in both hemispheres. The surface equivalent potential temperature $\bar{\theta}_{e s}$ in the bulk stability (7) is evaluated at the lowest model level. The convective bulk moist stability $\Delta_{e c}$ for $\gamma<1$ is calculated similarly to the convective bulk dry stability (see footnote 3 ), as the difference between the saturation equivalent potential temperature associated with the profile $\bar{T}_{c}$ evaluated at the temporal- and zonal-mean tropopause and the temporal- and zonal-mean equivalent potential temperature at the lowest model level. Equivalent potential temperatures are calculated using Bolton's (1980) formula. Absolute values of the convective bulk moist stabilities calculated in this way for $\gamma=1$ are less than $0.6 \mathrm{~K}$ for $\alpha \leq 2$ and do not exceed $3.1 \mathrm{~K}$ in any simulation, indicating that approximation and discretization errors are negligible for our purposes.
}

There is some arbitrariness in identifying the tropopause, as we did, with a $2 \mathrm{~K} \mathrm{~km}^{-1}$ isoline of temperature lapse rate. For example, this is generally not adequate in radiative-convective equilibrium (Fig. 3). Uncertainty in the identification of the tropopause can affect the bulk moist stability between the surface and the tropopause because the thermal stratification near the tropopause is considerably more stable than moist adiabatic (Fig. 2). Therefore, we also test whether the estimate (9) is consistent with the variations of the bulk moist stability between the surface and a fixed uppertropospheric level (cf. Frierson 2006, 2008). The tropopause plays no distinguished role in the arguments above, except as an upper bound on the height up to which convection can extend. If the arguments are sound, they should also be applicable to bulk moist stabilities between the surface and any fixed uppertropospheric level.

Figure 7 shows the extratropical bulk moist stability (7) between the surface and the fixed level $\sigma=0.4$, which lies below the extratropical tropopause in all but the coldest simulations with $\alpha=0.4$ (see Fig. 1). ${ }^{7}$ The new constant $d L_{e}=0.25 a$ used in this figure is again estimated to minimize the squared deviations of the bulk moist stabilities from the estimate (9) for the series of simulations with $\gamma=1$, excluding the simulation with $\alpha=0.4$. It is evident that the estimate (9) also is not consistent with these bulk moist stability variations. The bulk moist stability between the surface and the level $\sigma=0.4$ generally decreases with increasing $\alpha$, showing that the increase in the bulk moist stability up to the tropopause (Fig. 6) is primarily caused by an increase in tropopause height. However, particularly in warm climates (e.g., $\alpha \geq 2.5$ for $\gamma=1$ ), the equivalent potential temperature gradient at the lowest model level increases with $\alpha$ (Fig. 7a). The bulk moist stability variations implied by the estimate (9) for $\gamma=1$ thus have the opposite sign of the actual variations. In colder and moderately warm climates, the bulk moist stability varies without corresponding variations implied by the estimate (9), and including the convective bulk moist stability $\Delta_{e c}>0$ for $\gamma<1$ in the estimate (9) again leads to overestimation of bulk moist stability variations as $\gamma$ is varied (Fig. 7b).

The estimate (9) is not consistent with bulk moist stability variations in any climate regime encompassed

\footnotetext{
${ }^{7}$ The quantities in Fig. 7 are calculated analogously to those in Fig. 6 (see footnote 6). Absolute values of the convective bulk moist stabilities $\Delta_{e c}$ up to $\sigma=0.4$ calculated for $\gamma=1$ are less than $0.6 \mathrm{~K}$ for $\alpha \leq 2$ and do not exceed $2.6 \mathrm{~K}$ in any simulation, indicating that approximation and discretization errors are again negligible for our purposes.
} 

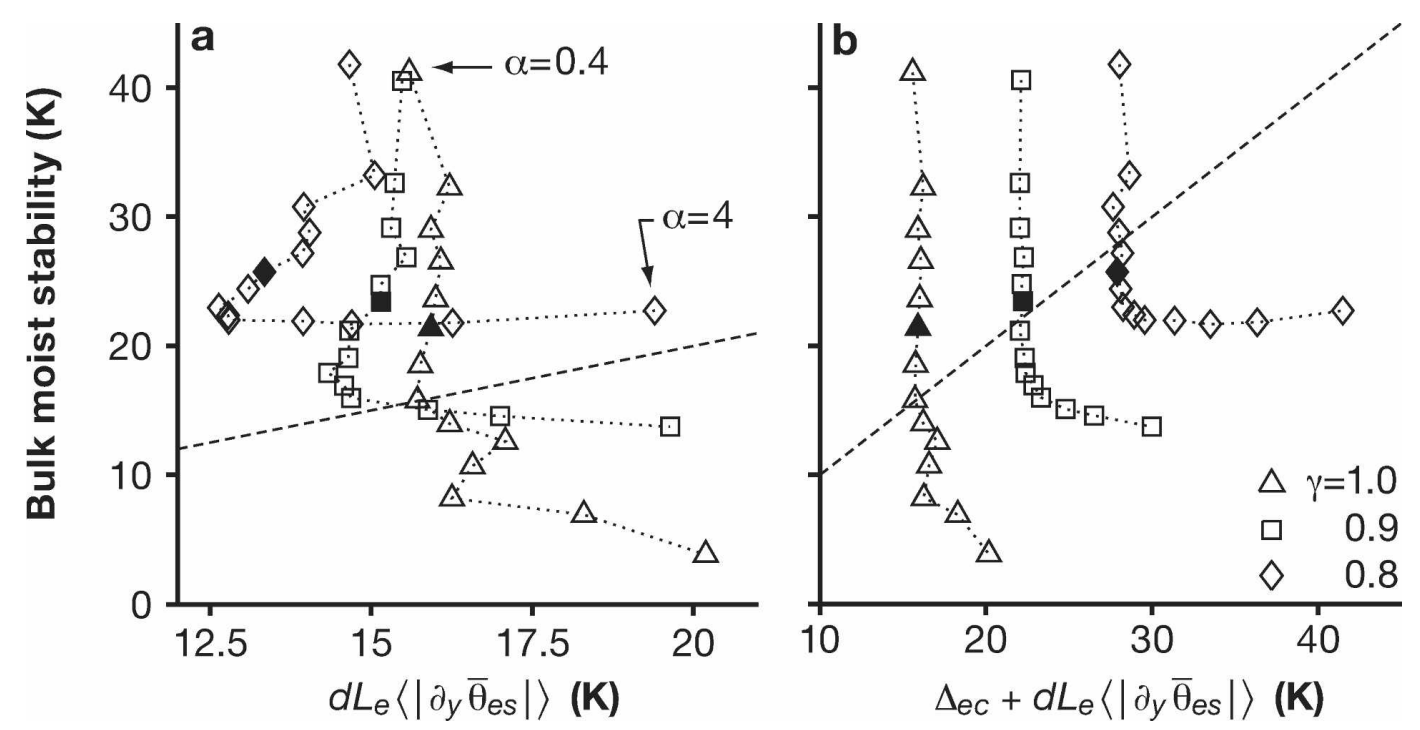

FIG. 7. Extratropical bulk moist stability between surface and the level $\sigma=0.4$ vs (a) $d L_{e}\left\langle\left|\partial_{y} \bar{\theta}_{e s}\right|\right\rangle$ and (b) $\Delta_{e c}+$ $d L_{e}\left\langle\left|\partial_{y} \bar{\theta}_{e s}\right|\right\rangle$, with $d L_{e}=0.25 a$. As in Fig. 6 , the results for $\gamma=1$ in (a) and (b) are identical because $\Delta_{e c} \equiv 0$, the dashed lines are the unit diagonals, and filled symbols identify the simulations with $\alpha=1$.

by the simulations. Different averaging conventions do not change this conclusion. For example, averaging over different latitude zones, which may be flowdependent as in SW06 or Frierson (2008), or using equivalent potential temperature gradients at higher levels in place of at the lowest model level does not substantially improve agreement of the simulation results with the estimate (9). As mentioned above, the results are sensitive to the level at which the equivalent potential temperature gradient is evaluated. For example, evaluating it not at the lowest model level but at the near-surface level $\sigma=0.89$ (roughly the level of maximum equivalent potential temperature variance in the lower troposphere and thus a justifiable choice) leads to poor fits of the estimate (9) to the simulation results: the equivalent potential temperature gradient at that level varies little with $\alpha$ over the entire range of climates simulated. Evaluating it in the midtroposphere, as in Juckes (2000), leads to a somewhat better fit of the estimate (9) to the bulk moist stabilities between the surface and the tropopause for the simulations with $\gamma=1$ because the equivalent potential temperature gradient in the midtroposphere increases over a wider range of climates than near the surface, as does the bulk moist stability. However, this likewise is not adequate over the entire range of climates simulated, and taking the variations of the convective lapse rate with $\gamma$ into account by including the convective bulk moist stability $\Delta_{e c}>0$ for $\gamma<1$ then leads to strong overestimation of the effect of the convective lapse rate on the bulk moist stability. Similarly, evaluating bulk moist stabilities between the surface and a different fixed upper-tropospheric level does not substantially alter the results in Fig. 7.

Only making assumption (i) above but not assumption (ii), and using $\left\langle\operatorname{std}\left(\theta_{e s}\right)\right\rangle$ as a proxy for $\left\langle\operatorname{std}\left(\theta_{e t}-\theta_{e s}\right)\right\rangle$ in the estimate (8), as in Frierson et al. (2006), likewise does not lead to an estimate of the bulk moist stability that is consistent with the simulation results. The standard deviation of equivalent potential temperature fluctuations near the surface is not generally proportional to the equivalent potential temperature gradient, even though eddy length scale variations are minimal. Using the standard deviation $\left\langle\operatorname{std}\left(\theta_{e s}\right)\right\rangle$ in place of $d L_{e}\left\langle\left|\partial_{y} \bar{\theta}_{e s}\right|\right\rangle$ gives a somewhat better fit to the bulk moist stabilities between the surface and the tropopause because, unlike the near-surface equivalent potential temperature gradient, the standard deviation generally (with few exceptions) increases with $\alpha$. However, the relation between bulk moist stabilities and standard deviations is not linear over the entire range of climates simulated, the variations of the bulk moist stability with convective lapse rate are still not captured, and the results are again sensitive to the level at which the standard deviation of equivalent potential temperature fluctuations is evaluated. For bulk moist stabilities between the surface and a fixed uppertropospheric level, the lack of fit seen in Fig. 7 remains. For example, although the standard deviation of equivalent potential temperature fluctuations near the surface generally increases with increasing $\alpha$, the bulk moist stability between the surface and a fixed upper- 
tropospheric level generally decreases. No linear relation involving convective bulk moist stabilities and equivalent potential temperature gradients or standard deviations appears to capture the bulk moist stabilities variations in the simulations.

Our results show that the agreement Frierson et al. (2006) and Frierson (2006, 2008) found with the bulk moist stability estimate (9) and variants thereof cannot generally be expected. The significant variations in the empirical constant $d$ among different series of simulations and observations examined by Frierson et al. also point to this lack of generality. Our variations of the convective lapse rate and the fact that bulk moist stabilities between the surface and the tropopause and between the surface and a fixed upper-tropospheric level can vary in opposite directions provide more stringent tests of the estimate (9) and variants thereof than those performed by Frierson et al.

\section{Conclusions}

Varying the lapse rate toward which parameterized moist convection in an aquaplanet GCM relaxes atmospheric temperature profiles has shown that moist convection only marginally affects the extratropical thermal stratification in Earth-like and colder climates. In warmer climates, moist convection does affect the extratropical thermal stratification, but the effect is still smaller than would be expected if moist convection alone controlled the thermal stratification. Because the quasi-equilibrium convection scheme in the GCM maintains a minimum static stability corresponding to the convective lapse rate, moist convection on the model's grid scale is suppressed in the simulations with reduced convective lapse rate and hence with a minimum static stability greater than that of a moist adiabat. Therefore, convection on the model's grid scale is also unlikely to affect the extratropical thermal stratification in climates in which the parameterized convection does not affect it significantly. Over the entire range of climates we simulated, including warm climates (globalmean surface air temperature up to $311 \mathrm{~K}$ ) with relatively small meridional surface temperature gradients (pole-equator temperature contrast as low as $25 \mathrm{~K}$ ), large-scale eddies modify the extratropical thermal stratification through their poleward and upward transport of sensible and latent heat and the release of latent heat in large-scale condensation.

Our findings are consistent with Earth's extratropical atmosphere in the zonal mean being more stably stratified than a moist adiabat. However, they contrast with the observation that extratropical air masses, for example, over midlatitude continents in summer and in storm tracks over oceans in winter, frequently exhibit thermal stratifications that are neutral with respect to moist convection or slantwise moist convection (Korty and Schneider 2007). In such regions, it appears that land-sea contrasts (e.g., moist maritime air advected over warm continents in summer) are crucial for moist convection to be more important for the extratropical thermal stratification than it is in our aquaplanet GCM simulations.

Theories and heuristics that have been proposed to account for either the thermal stratification (potential temperature structure) of dry atmospheres or for the thermal and moisture stratification (equivalent potential temperature structure) of moist-convecting atmospheres are not consistent with the simulation results. The estimate of SW06 of the pressure range over which large-scale eddy fluxes of dry entropy extend and the associated estimate of a dry bulk stability if eddies extend to the tropopause appear to be adequate in colder climates but are inadequate in warmer climates. The moist bulk stability estimate of Juckes (2000) and variants advocated by Frierson et al. (2006) and Frierson $(2006,2008)$ are not consistent with the simulation results. To account for the thermal stratification of moist atmospheres, it is essential to take latent heat release in phase changes of water into account-for example, in large-scale condensation in ascent regions of extratropical eddies-but moist convection may only need to be taken into account regionally and in sufficiently warm climates. Finding estimates of the thermal stratification that account for our and similar simulation results remains as a challenge to theorists.

Acknowledgments. We are grateful for support by the National Science Foundation (Grant ATM0450059) and by a David and Lucile Packard Fellowship. The simulations were performed on Caltech's Division of Geological and Planetary Sciences Dell cluster. Both the program code for the simulations, based on the Flexible Modeling System of the Geophysical Fluid Dynamics Laboratory, and the simulation results themselves are available from the authors upon request.

\section{REFERENCES}

Bolton, D., 1980: The computation of equivalent potential temperature. Mon. Wea. Rev., 108, 1046-1053.

Emanuel, K. A., 1988: Observational evidence of slantwise convective adjustment. Mon. Wea. Rev., 116, 1805-1816.

, J. D. Neelin, and C. S. Bretherton, 1994: On large-scale circulations in convecting atmospheres. Quart. J. Roy. Meteor. Soc., 120, 1111-1143.

Frierson, D. M. W., 2006: Robust increases in midlatitude static 
stability in simulations of global warming. Geophys. Res. Lett., 33, L24816, doi:10.1029/2006GL027504.

, 2007: The dynamics of idealized convection schemes and their effect on the zonally averaged tropical circulation. $J$. Atmos. Sci., 64, 1959-1976.

_ 2008: Midlatitude static stability in simple and comprehensive general circulation models. J. Atmos. Sci., 65, 1049-1062.

—, I. M. Held, and P. Zurita-Gotor, 2006: A gray-radiation aquaplanet moist GCM. Part I: Static stability and eddy scale. J. Atmos. Sci., 63, 2548-2566.

Held, I. M., 1982: On the height of the tropopause and the static stability of the troposphere. J. Atmos. Sci., 39, 412-417.

—, and B. J. Soden, 2000: Water vapor feedback and global warming. Annu. Rev. Energy Environ., 25, 441-475.

Juckes, M. N., 2000: The static stability of the midlatitude troposphere: The relevance of moisture. J. Atmos. Sci., 57, 30503057.

Korty, R. L., and T. Schneider, 2007: A climatology of the tropospheric thermal stratification using saturation potential vorticity. J. Climate, 20, 5977-5991.

O'Gorman, P. A., and T. Schneider, 2008a: The hydrological cycle over a wide range of climates simulated with an idealized GCM. J. Climate, 21, 3815-3832.

— and — 2008b: Energy of midlatitude transient eddies in idealized simulations of changed climates. J. Climate, 21, 5797-5806.

Schneider, T., 2004: The tropopause and the thermal stratification in the extratropics of a dry atmosphere. J. Atmos. Sci., 61, 1317-1340.

_ 2005: Zonal momentum balance, potential vorticity dynamics, and mass fluxes on near-surface isentropes. J. Atmos. Sci., 62, 1884-1900.

, 2007: The thermal stratification of the extratropical troposphere. The Global Circulation of the Atmosphere, T. Schneider and A. H. Sobel, Eds., Princeton University Press, 47-77.

— macroturbulence into critical states of weak nonlinear eddyeddy interactions. J. Atmos. Sci., 63, 1569-1586.

- , and 2008: Scaling laws and regime transitions of macroturbulence in dry atmospheres. J. Atmos. Sci., 65, 2153 2173.

Stone, P. H., 1972: A simplified radiative-dynamical model for the static stability of rotating atmospheres. J. Atmos. Sci., 29, 405-418.

—, 1978: Baroclinic adjustment. J. Atmos. Sci., 35, 561-571.

_- and J. H. Carlson, 1979: Atmospheric lapse rate regimes and their parameterization. J. Atmos. Sci., 36, 415-423.

Thuburn, J., and G. C. Craig, 2000: Stratospheric influence on tropopause height: The radiative constraint. J. Atmos. Sci., 57, 17-28.

Xu, K.-M., and K. A. Emanuel, 1989: Is the tropical atmosphere conditionally unstable? Mon. Wea. Rev., 117, 1471-1479. 